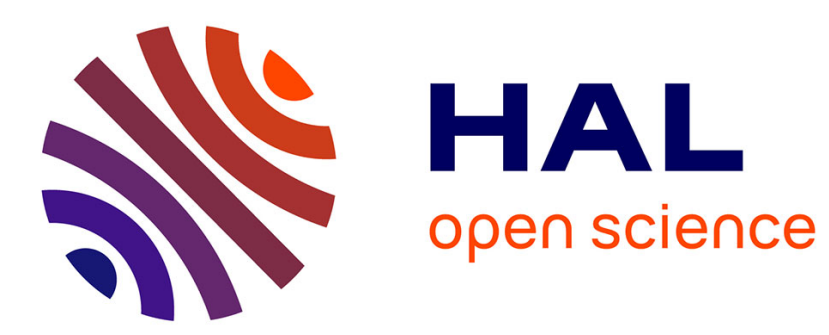

\title{
Referential and General Calls in Primate Semantics
}

Shane Steinert-Threlkeld, Philippe Schlenker, Emmanuel Chemla

\section{To cite this version:}

Shane Steinert-Threlkeld, Philippe Schlenker, Emmanuel Chemla. Referential and General Calls in Primate Semantics. Linguistics \& Philosophy, 2021. hal-03102596

\section{HAL Id: hal-03102596 https://hal.science/hal-03102596}

Submitted on 7 Jan 2021

HAL is a multi-disciplinary open access archive for the deposit and dissemination of scientific research documents, whether they are published or not. The documents may come from teaching and research institutions in France or abroad, or from public or private research centers.
L'archive ouverte pluridisciplinaire HAL, est destinée au dépôt et à la diffusion de documents scientifiques de niveau recherche, publiés ou non, émanant des établissements d'enseignement et de recherche français ou étrangers, des laboratoires publics ou privés. 


\title{
Referential and General Calls in Primate Semantics
}

\author{
Shane Steinert-Threlkeld \\ Department of Linguistics \\ University of Washington \\ shanest@uw.edu \\ Philippe Schlenker \\ Institut Jean-Nicod (ENS - EHESS - CNRS), Ecole Normale Supérieure \\ PSL University \\ New York University \\ Emmanuel Chemla \\ Laboratoire de Sciences Cognitives et Psycholinguistique \\ Dept d'Etudes Cognitives \\ ENS, PSL University, EHESS, CNRS \\ Forthcoming in Linguistics and Philosophy
}

\begin{abstract}
In recent years, the methods of formal semantics and pragmatics have been fruitfully applied to the analysis of primate communication systems. Most analyses therein appeal to a division of labor between semantics and pragmatics which has the following three features: (F1) calls are given referential meanings (they provide information about the world rather than just about an action to be taken), (F2) some calls have a general meaning, and (F3) the meanings of calls in context are enriched by competition with more informative calls, along the lines of scalar implicatures. In this paper, we develop highly simplified models to independently assess the conditions under which such features would emerge. After identifying a sufficient condition for (F1), we find a range of conditions under which (F2) and (F3) are not evolutionarily stable, and discuss the consequences for both modeling and empirical work.
\end{abstract}

In "Formal Monkey Linguistics" and "What Do Monkey Calls Mean?", Schlenker et al. [2016a,c] present three case studies in which they apply methods from formal linguistics to the analysis of monkey alarm calls.

Throughout the analyses, careful attention was paid to the division of labor between syntax, semantics, and pragmatics. All of the final analyses appeal to a division of labor between semantics and pragmatics which has the following three features: (F1) calls are given referential meanings (they provide information about the world rather than just about an action to be taken), (F2) some calls have a general meaning, and (F3) the meanings of calls in context are enriched by competition with more informative calls, along the lines of scalar implicatures. That the call systems of many different species 
can be given an analysis of a uniform type provides some motivation for the postulation of these three features. Nevertheless, because these features are not trivial, and some of them are thought to be unique to human language, some theorists have voiced resistance to their postulation. ${ }^{1}$

The present paper will provide independent arguments for all three features of these analyses. Our methodology throughout consists in developing highly simplified models in which one can observe the desired feature. These models do not constitute an analysis of a particular call system. Rather, their simplicity and resemblance to those in the evolution of signaling literature make it plausible that call systems with these three features could have arisen by standard evolutionary means, in other parts of the tree of life than the Homo genus.

The paper is structured as follows. In Section 1, we discuss each of these properties in greater detail and summarize some case studies that rely on them. The following sections take up, in turn, the three features of interest. Section 2 discusses the postulation of referential calls, i.e. (F1). The model developed there allows us to show the importance of conditions in which the receiver has information that the sender does not. We show how such asymmetries have been obfuscated in the existing literature but arise naturally in the environmental context of many primate species. Section 3 discusses the postulation of referential calls with general meanings and of competition between calls lying on a scale of informativity, i.e. (F2) and (F3). In particular, we develop a simple signaling model with a parameter controlling how optional competition based reasoning is. We find that under several variations of the basic game, optional competition can not be evolutionarily stable. After discussing the empirical and theoretical consequences of these results, we conclude in Section 4 with a brief discussion of future empirical and theoretical directions.

\section{Referential and general calls: motivations}

\section{$1.1 \quad$ Referential calls}

It is a standard assumption in the literature on primate communication that some monkey calls convey information about specific dangers. Thus a long line of research, following pioneering work by Seyfarth and Cheney, ${ }^{2}$ has shown by way of field experiments that (i) some calls are specifically triggered by the presence of some predators, such as eagles, and that (ii) conspecifics understand this information: when they hear an eagle-related monkey call, they adopt an eagle-appropriate reaction.

While one could take alarm calls to trigger specific reactions (like imperative meanings) rather than to convey information about the world (like propositional meanings), Seyfarth et al. [1980b] already provided data suggesting that this is sometimes the wrong way to go. For instance, a vervet monkey in a tree might react to a leopard-related call by looking down, whereas a monkey on the ground would not do so. In other words, conspecific reaction depends not just on the calls produced but also on the situation of the receiver. A simple imperative-based analysis would have difficulty accounting for this fact, whereas a referential analysis is more promising.

Zuberbühler et al. [1999] further highlight the referential nature of some calls by showing that there is a kind of 'conceptual equivalence' in Diana monkeys between an eagle shriek and an eagle-related conspecific call. As a baseline, the authors note that the

\footnotetext{
${ }^{1}$ See, among others, Jäger [2016], Steinert-Threlkeld [2016].

${ }^{2}$ See Seyfarth et al. [1980b,a].
} 
monkeys dishabituate (i.e. react less strongly) to an eagle shriek if it is preceded by an earlier eagle shriek — which is unsurprising since the second occurrence provides information that the monkeys already have. Crucially, the same dishabituation also occurs if the initial shriek is replaced with an eagle-related Diana call, which thus seems to convey to conspecifics the same kind of information as a shriek. On the other hand, dishabituation fails to occur to the same degree if the initial eagle-related call is replaced with a leopardrelated call. This is expected if dishabituation is due to the semantic content of the call rather than to the mere existence of an alert. ${ }^{3}$

\subsection{General calls and call competition}

\subsubsection{The Informativity Principle}

While initial studies highlighted the existence of calls with a narrow semantic content singling out specific predator classes, it was soon observed that many calls have more diverse uses, and may also elicit different reactions depending on the context. While this was initially taken to be a problem for 'referential' analyses of monkey calls (Macedonia and Evans [1993], Wheeler and Fischer [2012]), recent formal studies of primate semantics have embraced the existence of calls of different levels of specificity (Schlenker et al. [2016c]; see also Dezecache and Berthet [2018] for a survey of the literature and facts about general calls). Thus a call may indicate eagle presence, while another call may just raise an alert without specifying its precise nature.

Importantly, the use of specific and more general calls has been argued to be regulated by an 'Informativity Principle' that mandates that the most specific call compatible with a situation should be used, as stated in (1).

\section{The Informativity Principle}

If the sender produced a call $\mathrm{C}$ which evokes ('competes with') a call C', if $\mathrm{C}$ ' is more informative than $\mathrm{C}$, infer that $\mathrm{C}$ ' is false (for if $\mathrm{C}$ ' were true the sender should have uttered it).

In human language, a similar principle is responsible for so-called 'implicatures' (Grice [1975]). From the sentence 'it is possible that there's traffic on the route', one typically infers that the speaker is not in a position to assert that 'it is certain that there's traffic on the route', which would have been more informative. This is due to the competition between 'certain' and 'possible', combined with a human version of the Informativity Principle. In linguistics, this principle is taken to derive from cooperative principles of language use, and in particular from the existence of a sophisticated theory of mind: speakers try to be as informative as possible (Grice [1975], but see Chierchia [2013], Chierchia et al. [2012] for a slightly different view). Schlenker et al. [2016a], however, go to great lengths to emphasize that the primate version of the Informativity Principle need not be committed to the existence of a sophisticated primate theory of mind (nor to sophisticated grammatical abilities): competition may be hard-wired, or just follow from far weaker principles.

\footnotetext{
${ }^{3}$ Note that one might attempt to explain these facts by positing that animals do not tend to repeat actions. Zuberbühler et al. [1999] (p. 39) consider this hypothesis, but argue that it makes predictions that are not supported by the data. See Suzuki [2018] for a similar argument about repetitions in birds.
} 


\subsubsection{Putty-nosed monkey calls}

The general motivation for the existence of general calls and for the Informativity Principle can be illustrated by the example of Putty-nosed monkeys. They have a hack call which is usually eagle-related, and a pyow call which is used in leopard-related situations but also in all sorts of other situations. Hence, pyow seems to be used in a set of situations that do not form a 'natural class'. Schlenker et al. [2017] posit, however, that its meaning corresponds to the existence of an alert, which defines a natural class of situations, but that the competition with hack is responsible for its restriction to non-eagle-related situations (and thus for the fact that its extension does not appear to be a 'natural class', but a natural class with a hole, so to speak). Specifically, they posit meanings akin to (2) (we have slightly simplified their presentation).

(2) Meaning of Putty-nosed calls

a. $\llbracket p y o w \rrbracket=1$ iff there is an alert

b. $\quad \llbracket h a c k \rrbracket=1$ iff there is a serious non-ground, movement-related alert

As one would expect, one finds pyow in leopard-related situations. One also finds pyow at the end of sequences produced in eagle-related situations, which is still compatible with its very general meaning. However one needs to explain why one almost never finds pyow at the beginning of eagle-related sequences.

Schlenker et al. [2016a, 2017] take the explanation to lie in the Informativity Principle: soon after an eagle alert, there is a serious non-ground, movement-related alert, and thus hack can be used. By the Informativity Principle, it trumps pyow, which is less informative. Things are different at the end of eagle-related sequences, presumably because the level of danger gradually weakens after an alert, with the result that at some point hack, which is indicative of serious alerts, cannot be used any more.

It should be noted that this analysis could be emulated without general calls and without an Informativity Principle if one were willing to make two assumptions: first, that pyow is specified as an alert call used in situations that are not serious, non-ground related ones (what Schlenker et al. [2016a] took to be a non-natural class); and second, that competition occurs without exception, so that the outcome of the Informativity Principle is to make the extension of pyow the complement of the extension of hack within alert situations. The first assumption may lead one to produce very unnatural meanings, but for lack of a general theory of what 'natural meanings' are, this point is somewhat speculative (see however, e.g., Gärdenfors [2000], Chemla et al. [2019] for discussions as to why 'holes' may be unnatural, in human and non-human meanings, respectively). The second assumption seems to be too strong, as we will see in other cases below-and this will play an essential role in our formal discussion.

It is worth noting that Schlenker et al. [2017, 2016a] posit a further competition principle, the Urgency Principle, which mandates that calls that provide information about the nature and location of a threat appear early in a sequence. The motivation for this principle lies in the behavior of so-called pyow-hack sequences, which trigger group movement in Putty-nosed monkeys. The authors show that this result can be derived on the basis of the weak meanings in (2), combined with the Urgency Principle. This is another instance of the strategy at work in the Informativity Principle: weak lexical meanings are posited, but are then enriched by way of pragmatic principles. 


\subsubsection{Titi monkey calls}

To highlight the generality of the phenomenon, let us briefly turn to the calls of Titi monkeys, which also motivate an analysis with general calls and an Informativity Principle. Black-fronted Titi monkeys (Callicebus nigrifrons, South America) exhibit a seemingly complex call system whereby a sequence of calls of just two types can encode information about both type and location of various predators. Cäsar et al. [2013] report the results of field experiments in which model predators were placed either in the canopy or on the ground to elicit call sequences. Simplifying somewhat, the data to be explained can be summarized as in (3) (here $A^{+}$refers to a series of A-calls and $B^{+}$refers to a series of B-calls):

\section{Main generalizations}
a. Raptor, canopy: $A^{+}$
b. Raptor, ground: $A^{+} B^{+}$
c. Cat, canopy: $A B^{+}$
d. Cat, ground: $B^{+}$
e. Non-predation: $B^{+}$

Schlenker et al. [2016c] argue that a non-compositional analysis in which entire sequences are interpreted as units lacks plausibility, in part because of the sheer time it would take to decide whether one message or another is being conveyed (the sequences can be very long). They also suggest that the appearance of a complex syntax is an illusion: they provide an analysis in which each call is a separate utterance (hence they are no syntactic or semantic combination rules), and it is the interaction between simple meanings, context change and world knowledge that yields fairly stereotyped sequences. The meanings they posit are given in (4); they are relativized to a time of utterance because context change plays a crucial role in the analysis:

(4) Meaning of Titi calls

a. $\llbracket B \rrbracket^{t}=1$ iff there is a noteworthy event at $t$

b. $\llbracket A \rrbracket^{t}=1$ iff there is a serious non-ground alert at $t^{4}$

For our purposes, the important observation is that the B-call appears in extremely diverse situations, pertaining to predation and non-predation alike. The authors take it to be a general call. But crucially, in an eagle-related situation, although the B-call could be used, it never appears at the beginning of a sequence. This is the same situation that we already discussed in connection with the Putty-nosed pyow call, and the authors' solution is the same: the B-call is indeed general, but its use is constrained by the Informativity Principle, which mandates that the (more informative) A-call should be produced whenever possible. ${ }^{5}$

\subsubsection{Campbell's calls}

A particularly non-trivial use has been made of the Informativity Principle in the analysis of Campbell's communication, which involves calls slightly differently on two sites, the Tai forest in Ivory coast and Tiwai island in Sierra Leone. While the two monkey populations

\footnotetext{
${ }^{4}$ Commier and Berthet [2019] argue that the 'non-ground' aspect of this meaning is redundant. Because nothing in the present paper hinges on this issue, we present the richer meaning.

${ }^{5}$ See Berthet et al. [2018, 2019] for more recent and complicated data on Titi calls.
} 
might not be very different genetically, they don't have the same predators: the Tai monkeys are prey to leopards or eagles, whereas the Tiwai monkeys only have to deal with eagles [Ouattara et al., 2009].

In the Tai forest, male adults have a non-predation-related call, boom. They use krak to raise leopard alerts, and hok for raptor alerts. They also have suffixed calls: $k r a k$ oo is used for unspecific alerts, and hok-oo for non-ground disturbances. The challenge is to assign meanings to boom, krak, hok, and -oo. But further complexity is added by Campbell's call use on Tiwai Island, where leopards haven't been seen for decades: the Tai calls are used, but krak raises unspecific alerts (as does krak-oo), rather than leopard alerts.

Simplifying somewhat, the authors posit for both sites the meanings in (5).

Meaning of Campbell's calls

a. $\llbracket k r a k \rrbracket=1$ iff there is a disturbance

b. $\llbracket h o k \rrbracket=1$ iff there is a non-ground disturbance

c. $\llbracket b o o m \rrbracket=1$ iff there is a disturbance but no predator

d. for any root $\mathrm{R}$ except boom-boom, $\llbracket R-o o \rrbracket=1$ iff there is a disturbance that licenses $\mathrm{R}$ and isn't strong among disturbances that license $\mathrm{R}$.

The authors make crucial use of the Informativity Principle. First, hok competes with other calls, and because hok-oo (pertaining to weak non-ground alerts rather than to any non-ground alert) is more specific, the meaning of hok is enriched to something like hok but not hok-oo: it only applies to aerial (hok) non-weak (not hok-oo) alerts - which explains the raptor uses. Similarly, the unspecific alert krak competes with krak-oo, but also with hok. Due to this double competition, in the end krak can only be used for serious (not krak-oo) ground (not hok) disturbances. This accounts for the leopard uses observed in Tai. Schlenker et al. [2014] further note that in the Tai forest krak is used more often than one would expect if it is a leopard call: there seem to be residual 'unenriched' uses of this call. This suggests that the Informativity Principle does not apply completely systematically (although data on this point are very preliminary).

Finally, the authors seek to explain why krak has a different use on Tiwai island, where it raises unspecific alerts. Importantly, this use just corresponds to the basic (unenriched) meaning of krak. The authors posit that this bare meaning fails to be pragmatically enriched on Tiwai because enrichment would yield a useless meaning for lack of serious ground predators. Without the pragmatic enrichment, we are left with the literal and general meaning of $k r a k$ on Tiwai island.

\subsection{Summary and outlook}

The analyses of Putty-nosed, Titi and Campbell's calls all rely on the three features discussed in the introduction.

(F1) The calls have referential meanings: in all of the analyses, the meanings are defined truth-conditionally by specifying what external environmental condition must obtain to license the call. Thus these meanings provide information about the world, rather than directly specifying an action to be taken.

(F2) The meanings of calls come in different levels of specificity: pyow is more general than hack in Putty-nosed monkeys, the B-call is more general than the A-call in Titi monkeys, and krak is more general than hok in Campbell's monkeys. 
(F3) The meanings of general calls are enriched by competition with more informative calls: this is captured by the Informativity Principle (1), which was crucially used in all three case studies, and derived particularly non-trivial patterns in Campbell's monkeys.

We now turn to the task of explaining how call systems with each of these three features could have arisen. We discuss reasons why one may think these features should not have arisen, and present simple models to explore these reasons. For (F1), we identify a sufficient condition. For (F2) and (F3), our modeling expands the breadth of the worry about their presence, but points the way forward for alternative explanations.

\section{Referential Meanings}

Turning to the first property (F1), we ask: what can justify positing referential meanings, as opposed to action-based meanings? There are at least two reasons to be skeptical of referential meanings. On the one hand, growing out of the literature on functional reference, there appears to be a certain symmetry between the cause of a call and the actions it causes. Consider the paradigm case: vervet monkey calls (see Seyfarth et al. [1980a]). They have three predators, and tend to emit different alarm calls in the presence of each, which in turn tend to elicit adaptive behaviors in the receivers of the calls. ${ }^{6}$ Each call is then said to functionally refer to the associated predator. But there seems to be no reason to favor a meaning for the call that places primacy on the referential side or on the induced action side. This is why Macedonia and Evans [1993] have a 'production' and a 'perception' criterion in their definition of functional reference. ${ }^{7}$ Why, then, provide a semantic analysis of alarm calls solely in terms of reference? ${ }^{8}$ Cases in which one and the same call triggers different reactions depending on the environmental context and/or on the receiver's situation would be crucial, as we mentioned at the outset. While data do exist, as we mentioned above in relation to vervet monkeys, they are not overwhelming yet.

On the other hand, it is unclear how calls with a referential meaning can be the units of selection in evolution. In standard models of the evolution of alarm calls, ${ }^{9}$ fitness of both sender and receiver is determined by the action taken by the receiver, and its consequences. Because of this, referential meanings are not directly relevant for fitness. Why, then, posit such meanings in the first place? Jäger [2016] puts the point especially forcefully (p. 114): "This begs the question how [meanings besides the receiver's action] - and therefore the distinction between semantics and pragmatics - could have evolved in the first place in connection with innate signaling systems." While the full answer to this challenge overlaps with properties (ii) and (iii), justifying property (i) is a necessary step along the way.

In the rest of this section, we present a generalization of signaling games $(\S 2.1)$ and provide a definition of the conditions in which an action-based analysis of a system can be given. In order to explicate Jäger's objection, we will provide a sufficient condition

\footnotetext{
${ }^{6}$ See Price et al. [2015] for evidence of wider distribution of Vervet calls than merely in alarm contexts.

${ }^{7}$ The more refined definitions in Scarantino [2013], Scarantino and Clay [2015] still have this dual character.

${ }^{8}$ This issue has also arisen in the signaling games literature. Lewis [1969] already noticed that he cannot distinguish between assertions and imperatives, because in his signaling systems there is no way to break the symmetry between cause of production of a signal and the action it then causes. Huttegger [2007] and Zollman [2011] provide models which attempt to break this symmetry. We discuss them more below. Skyrms [2010] shows how to quantify the amount of information of each type.

${ }^{9}$ See, for an overview, Skyrms [2010].
} 
on being able to do so $(\S 2.2)$. In particular, if the speaker is as-or-more-informed than the audience and communicates all of its information, then an action-based analysis can be given. We then present simple scenarios where an action-based analysis cannot be given, illustrating failures of each of the jointly sufficient conditions. We also show how the presence of multiple receivers can lead to either condition failing, explicating some observations in the literature arguing that the presence of multiple receivers precludes an action-based analysis ( $(2.3)$. Our result shows that when the sender is strictly more informed than the receiver, it is impossible to distinguish referential analyses from actionbased analyses. This fully justifies Jäger's objection in typical conditions, and also shows how it may be alleviated in the general case. We conclude (§ 2.4) by discussing an earlier proposal for distinguishing referential and action-based communication.

\subsection{The Model}

As just mentioned, our model generalizes standard signaling games. In particular, we need a richer conception of the information available to the sender and the receiver in order to capture more relationships between their information. The model has the following components:

- A space $W$ of worlds

- A probability distribution $P$ on $W$

- A sender $\sigma$ and a partition $\mathbf{S}$ of $W$, which represents $\sigma$ 's view of the world.

- A receiver $\rho$ and a partitions $\mathbf{R}$ of $W$, which represents $\rho$ 's view of the world.

- A set $M$ of messages.

- A set $A$ of actions that the receiver may undertake.

The main difference in the set-up concerns the partitions, reflecting the players' view of the world, and the signaling game itself will make use of these partitions. For a world $w \in W$ and a partition $\mathbf{P}$ of $W$, we will denote by $[w]_{\mathbf{P}}$ the cell of $\mathbf{P}$ that contains $w$. A play of the game runs as follows:

1. Nature chooses $w \in W$ with probability $P(w)$.

2. Nature informs the sender of $[w]_{\mathbf{S}}$ and the receiver of $[w]_{\mathbf{R}}$.

3. The sender chooses a message $m$, based on their view of the world, and sends it to the receiver.

Due to this behavior, we conceive of the sender $\sigma$ as a function from $\mathbf{S}$ to $M$.

4. The receiver chooses an action $a$, based on their view of the world and the message they received.

Due to this behavior, we conceive of the receiver $\rho$ as a function from $M \times \mathbf{R}$ to $A$.

The simplest type of signaling game makes the following choices: there is only one receiver, ${ }^{10} \mathbf{S}=\{\{w\}: w \in W\}$ is the finest partition of $W$ and $\mathbf{R}=\{W\}$ is the trivial partition of $W$. This captures a very strong information asymmetry: the sender is maximally

\footnotetext{
${ }^{10}$ Often in games with multiple receivers, the following information asymmetry is made, so the reader can prefix what follows with implicit quantification over the receivers.
} 
informed and the receiver is minimally informed. As we will see, a necessary condition on needing to give a referential analysis will be breaking this strong asymmetry: the sender will need to not be more informed than the receiver. The most natural scenarios will involve information incomparability, so that both the sender and the receiver have information that the other lacks. ${ }^{11}$

We note also that we are not presently incorporating a utility function into the model. We do this in order to distinguish two questions: (i) whether the analysis of some animal call behavior can be given in action-based terms, and (ii) whether the observed call behavior is in some sense optimal or adaptive. Our primary concern is question (i), though we will return to (ii) below. While the assumption of optimality / adaptiveness can help explain observed behavior, we are primarily concerned with the types of analysis of a given pattern of observed behavior, which is an orthogonal issue.

\subsection{A Condition for Referential Analyses}

Before moving on to examine the conditions for referential analyses, we need a couple of auxiliary definitions. A partition $\mathbf{P}$ refines a partition $\mathbf{P}^{\prime}$ iff every cell of $\mathbf{P}^{\prime}$ is a union of cells of $\mathbf{P}$. In this situation, we also say that $\mathbf{P}^{\prime}$ is a coarsening of $\mathbf{P}$. Note that by these definitions, every partition both refines and coarsens itself; strict versions of the concepts are gained by requiring the two partitions to be non-equal.

One can think of $\mathbf{P}$ refining $\mathbf{P}^{\prime}$ in the following way: $\mathbf{P}$ is more informative than $\mathbf{P}^{\prime}$, in that by locating oneself in a cell of $\mathbf{P}$, one thereby locates oneself in a cell of $\mathbf{P}^{\prime}$. If, as standard, ${ }^{12}$ we think of partitions as questions, whose cells are total answers, then answers to $\mathbf{P}$ are also answers to $\mathbf{P}^{\prime}$. By $\mathbf{P} \cap \mathbf{P}^{\prime}$, we will denote the partition obtained by taking the intersection of the equivalence relations corresponding to $\mathbf{P}$ and $\mathbf{P}^{\prime}$. Two worlds belong to the same cell of $\mathbf{P} \cap \mathbf{P}^{\prime}$ just in case they belong to the same cell in each of $\mathbf{P}$ and $\mathbf{P}^{\prime}$. We will make use of the following derived partitions:

- $\mathbf{M}:=\{[m]: m \in M\}$, where $[m]=\{w: \sigma(w)=m\}$ is the set of worlds in which the sender sends message $m$.

In other words: two worlds are in the same cell of $\mathbf{M}$ just in case the receiver gets the same message from the sender in each.

Note that, by definition, $\mathbf{M}$ coarsens $\mathbf{S}$ (which represents the sender's view of the world).

- $\mathbf{A}:=\{[a]: a \in A\}$, where $[a]=\{w: \rho(w)=a\}$ is the set of worlds in which the receiver undertakes action $a$.

In other words: two worlds are in the same cell of $\mathbf{A}$ just in case the receiver undertakes the same action in each.

Note that, by definition, $\mathbf{A}$ coarsens $\mathbf{M} \cap \mathbf{R}$.

With these helpers in place, we can articulate a definition of an action-based analysis for a call system or, skipping a step ahead, a condition on a call system when action-based meanings can be given to the calls.

\footnotetext{
${ }^{11}$ See Geanakoplos and Polemarchakis [1982], Goodman and Stuhlmüller [2013] for examples of signaling games without the aforementioned information asymmetry. The related field of epistemic game theory [Perea, 2012, Pacuit and Roy, 2015] studies explicitly agents' reasoning about each others' information.

${ }^{12}$ Following the pioneering work of Hamblin [1973], Groenendijk and Stokhof [1984].
} 
(R) An action-based analysis of the calls can be given if and only if $\mathbf{M}$ refines $\mathbf{A}$ (i.e. if and only if the information conveyed by the messages is as-or-more fine-grained than the information conveyed by the actions taken).

The rationale for $(\mathrm{R})$ runs as follows. Suppose that $\mathbf{M}$ refines $\mathbf{A}$. Then knowing which message was sent will suffice to know which action was taken. In such a scenario, one may analyze the meaning of each message as a command to take the action that it always causes the receiver to take. But this will then be an action-based analysis: so if $\mathbf{M}$ refines $\mathbf{A}$, then an action-based analysis can be given. Alternatively, suppose that an action-based analysis can be given. We take it that one should only give such an analysis if each message in the system wholly determines which action the receiver will take. ${ }^{13}$ But in that case, we will have that $\mathbf{M}$ refines $\mathbf{A}$, since no worlds that agree on the message sent can disagree on the action taken. Therefore, if an action-based analysis can be given, then $\mathbf{M}$ refines $\mathbf{A}$.

We now articulate some consequences of condition $(R)$. In particular, we note the following sufficient condition on being able to give an action-based analysis.

(6) If (i) the sender is as-or-more informed than the receiver ( $\mathbf{S}$ refines $\mathbf{R}$ ) and (ii) communicates all of its information to the receiver ( $\mathbf{M}$ equals $\mathbf{S}$ ), then an actionbased analysis can be given ( $\mathbf{M}$ refines $\mathbf{A}$, see $(R)$ ).

The proof of this observation is straightforward. As was noted above, $\mathbf{A}$ coarsens $\mathbf{M} \cap \mathbf{R}$. By the assumptions in the antecedent of (6), however, $\mathbf{M} \cap \mathbf{R}=\mathbf{M}$, so $\mathbf{A}$ coarsens $\mathbf{M}$, i.e. $\mathbf{M}$ refines $\mathbf{A} .^{14}$

An example will help illustrate the general structure. $W$ has four worlds $T L, T R$, $B L$, and $B R$ (think: top-left, top-right, et cetera). The sender has two messages. The receiver can tell what column obtains, so the partition $\mathbf{R}$ is what we will call column $:=$ $\{\{T L, B L\},\{T R, B R\}\}$. We assume that the receiver is 'observed' performing $a_{1}$ in states $T L$ and $B R$ and $a_{2}$ in states $T R$ and $B L$. There are at least two ways this behavior could arise.

Suppose the sender can tell what row obtains, so the partition $\mathbf{S}$ is what we will call row $:=\{\{T L, T R\},\{B L, B R\}\}$. Further suppose that the sender sends one message when it learns top row and the other when it learns bottom row. Thus we have that $\mathbf{M}=\mathbf{S}$. But: $\mathbf{M}$ does not refine $\mathbf{A}$, since there is variation in the action taken across the cells of $\mathbf{M}$. In this case, the receiver uses its partition column to make its choice of action. In this case, we clearly have that $\mathbf{S}$ does not refine $\mathbf{R}$, since learning the row is not more informative than learning the column. (In fact, these partitions have an even stronger property: they are orthogonal. But that stronger property is not necessary for referential analysis.)

Alternatively, we can suppose that $\mathbf{S}$ is the finest partition, but the sender still only

\footnotetext{
${ }^{13}$ By wholly determining the action, we intend to include only relatively 'physiologically simple' actions and to exclude conditional imperatives, indexicals, and the like from counting as determining an action.

${ }^{14}$ The reader may find the contrapositive statement and its proof to be helpful. If $\mathbf{M}$ does not refine $\mathbf{A}$, then either $\mathbf{M} \neq \mathbf{S}$ or $\mathbf{S}$ does not refine $\mathbf{R}$. Proof: Suppose that $\mathbf{M}$ does not refine $\mathbf{A}$. So: there is a cell $c_{a} \in \mathbf{A}$ such that $c_{a}$ is not the union of any set of cells from $\mathbf{M}$. Because $\mathbf{A}$ coarsens $\mathbf{R} \cap \mathbf{M}$, we have that $c_{a}$ is the union of a set of cells from the latter. That is to say: for all $\mathrm{m} \subseteq \mathbf{M}, c_{a} \neq \bigcup \mathrm{m}$, while at the same time there is $\mathrm{m} \subseteq \mathbf{M}, \mathrm{r} \subseteq \mathbf{R}$ such that $c_{a}=\bigcup\{m \cap r: m \in \mathrm{m}, r \in \mathrm{r}\}$. Together, these imply that for some $m \in \mathbf{m}, r \in \mathrm{r}, r \cap m \neq m$, which entails that $\mathbf{M}$ does not refine $\mathbf{R}$. Now: recall that $\mathbf{M}$ is a coarsening of $\mathbf{S}$. So, either $\mathbf{M}=\mathbf{S}$, in which case the above reasoning shows that $\mathbf{S}$ does not refine $\mathbf{R}$, or $\mathbf{M} \neq \mathbf{S}$.
} 
has two messages. The sender could do at least two things. It can send $m_{1}$ in $T R$ and $B L$ and $m_{2}$ in the other two states. In this case, we will have that $\mathbf{M}=\mathbf{A}$, so an action-based analysis could be given. Alternatively, the sender can still send $m_{1}$ in the top row and $m_{2}$ in the bottom row, relying on the receiver to use its additional information to make its choice. In this case, $\mathbf{M}$ does not refine $\mathbf{A}, \mathbf{S}$ does refine $\mathbf{R}$, but $\mathbf{M} \neq \mathbf{S}$.

\subsection{Multiple Receivers}

It has often been remarked that when there are multiple receivers, an action-based analysis cannot be given since different receivers may have to take different actions. This situation arises naturally in Campbell monkeys, which have groups in which the resident male issues alert calls to the rest of the group. ${ }^{15}$ Zollman [2011] reports that Brian Skyrms made exactly this suggestion: "Since the right response for a listener depends on the situation that listener is in - and since the signaler is signaling to many different monkeys in different contexts of which the signaler is unaware -it seems proper to interpret the signal as an assertion." We will now extend the model and result to handle multiple receivers and develop two simple analyses of this situation. In one case, it is indeed the sender's unawareness that makes it impossible to develop an action-based analysis. In another case, it is simply the lack of enough signals. This shows that, while the presence of multiple receivers can preclude action-based analyses, this can be so for different reasons, which are elucidated in (7) below.

In the extended model, there are now $n$ receivers $\rho_{1}, \ldots, \rho_{n}$, each with their own view of the world (i.e. partition) $\mathbf{R}_{1}, \ldots, \mathbf{R}_{n}$. For simplicity, we will assume that each receiver has the same set of available actions. Similarly, the sender can send a message from $M$ to each receiver, with distinct receivers possibly receiving distinct messages. We will denote the total message sent by $\sigma$ as $\vec{m}$ and the particular message sent to receiver $i$ by $\vec{m}_{i}$. The same notation applies, mutatis mutandis, to the $n$ actions $\vec{a}$ chosen by the receivers. $\mathbf{M}$ will denote the partition whose cells contain worlds in which the sender sends the same total message, i.e. worlds which agree on the message that the sender sends to each receiver. Similarly, A's cells contain worlds which agree on the action taken by all of the receivers. ${ }^{16}$ The generalization of (6) to the multiple-receiver situation is straight-forward:

If (i) the sender is as-or-more informed than each receiver ( $\mathbf{S}$ refines $\mathbf{R}_{i}$ for every i) and (ii) communicates all of its information $(\mathbf{M}=\mathbf{S})$, then an action-based analysis can be given ( $\mathbf{M}$ refines $\mathbf{A}$, see $(\mathrm{R})$ ).

Having generalized to multiple receivers, we can now model simple scenarios capturing Skyrms' insight from above. Suppose there are two receivers, each in one of two locations - the ground $(g)$ or the canopy $(c)$ - and two predators - eagles $(E)$ and leopards $(L)$. We can take the worlds in $W$ to be tuples consisting of a predator and two locations. As an example, we can model as $\langle E, c g\rangle$ the state in which there is an eagle present $(=E)$, while the first receiver is in the canopy $(=c)$ and the second receiver is on the ground $(=g)$. We can further suppose that each receiver will take an appropriate action given its location if it knows the predator (e.g. each will climb a tree in the presence of a predator

\footnotetext{
${ }^{15}$ See Zuberbühler [2001].

${ }^{16}$ More formally, $\mathbf{M}=\mathbf{M}_{1} \cap \cdots \cap \mathbf{M}_{n}$, where $\mathbf{M}_{i}=\left\{\left\{w: \overrightarrow{\sigma([w] \mathbf{S})_{i}}=m\right\}: m \in M\right\}$ is the partition where worlds belong to the same cell if the sender sent the same message to receiver $i$ in each world. And mutatis mutandis for $\mathbf{A}$ and $\mathbf{A}_{i}$.
} 
if they are on the ground). Let us suppose that the sender has two messages and that it must send the same message to both receivers:

(C) For every $s \in \mathbf{S}, \overrightarrow{\sigma(s)}_{1}=\overrightarrow{\sigma(s)}_{2}$

Case 1: the sender knows the predator, but not the locations. In this case, $\mathbf{S}$ is the partition that distinguishes eagle worlds (i.e. those with an $E$ in the first component) from leopard worlds (i.e. those with an $L$ in the first component), but lumps together all location combinations with the same predator. Because $(\mathrm{C})$ obtains, it seems that the best thing for the sender to do is send a distinct message in each cell, yielding $\mathbf{M}=\mathbf{S}$. We have, however, that $\mathbf{S}$ does not refine either $\mathbf{R}_{i}$, since each receiver knows its own location. And this is indeed a case where $\mathbf{M}$ does not refine $\mathbf{A}$, under the plausible assumption that the receivers take different actions in different locations in response to one of the predators.

Case 2: $\mathbf{S}$ is the finest partition. Nevertheless, because (C) obtains and there are only two messages, we will have $\mathbf{M} \neq \mathbf{S}$ in this case. And indeed $\mathbf{M}$ does not refine $\mathbf{A}$ under the same assumption about receivers' actions.

This little exercise shows that either of the jointly sufficient conditions on being able to give an action-based analysis ((i) and (ii) in (7)) may obtain without the other in the presence of multiple receivers. Moreover, the presence of multiple receivers does not necessarily preclude action-based analyses. Thus, while a situation with multiple receivers often does preclude action-based analysis, this can be so for different reasons. In the next section, we examine the models of Zollman [2011] in the present framework, showing that they allow an action-based analysis with different 'dialects'.

\subsection{An Earlier Analysis}

Zollman introduces a game and an equilibrium therein, which he took to illustrate assertions. The intuitive set-up is as follows: there are two agents carrying a couch and a third one talking to them. It is hard to keep the couch horizontal, and the couch thus tends to be in one of two states: tilted up or tilted down. If it is tilted up, the agent at the front should lower it and the person at the back should raise it. If it is tilted down, it is the other way around. In each state, the right thing for the agent at the front of the couch to do is the opposite of the right thing for the agent at the back of the couch to do. The sender can send a message to each lifter. Zollman models this as follows: $W=\{\mathrm{Up}$, Down $\}, \mathbf{S}=\{\{w\}: w \in W\}, \mathbf{R}_{1}=\mathbf{R}_{2}=\{W\}, M=\left\{m_{1}, m_{2}\right\}, A=\left\{a_{1}, a_{2}\right\}$, and $\vec{a}(\sigma(\mathrm{UP}))=\left\langle a_{1}, a_{2}\right\rangle, \vec{a}(\sigma(\mathrm{DowN}))=\left\langle a_{2}, a_{1}\right\rangle$. Zollman considers two cases:

$$
\begin{array}{ll}
\text { a. } & \sigma(\mathrm{UP})=\left\langle m_{1}, m_{2}\right\rangle, \sigma(\mathrm{Down})=\left\langle m_{2}, m_{1}\right\rangle . \\
\text { b. } & \sigma(\mathrm{UP})=\left\langle m_{1}, m_{1}\right\rangle, \sigma(\mathrm{DowN})=\left\langle m_{2}, m_{2}\right\rangle .
\end{array}
$$

He claims that in (8-a), the messages have a purely imperative content (e.g. $m_{1}$ means 'lower!') and that in (8-b), the messages have a purely indicative content (e.g. $m_{1}$ means 'the couch is tilted up').

In the present framework, we have that $\mathbf{M}$ does in fact refine $\mathbf{A}$ not just in (8-a), but also in (8-b). By (R), this means that an action-based analysis of the system can be given. A first pass would be a group command analysis: in (8-b), $m_{1}$ could be taken to be the group command 'flatten the couch out by lowering the front', and $m_{2}$ could be the opposite. We note, however, that for such an analysis to count as action-based, rather complex actions would have to count as primitive: while raising one's arms up 
may count as an 'action', it is a bit different to consider 'flattening the couch' as an action. This certainly would depart quite far from views in animal communication in which the reactions to calls are analyzed as more akin to physiological reflexes. While the group command gloss provided above does carry imperative mood, it does not count as action-based for our purposes.

Nevertheless, an action-based analysis can be given by making a stronger observation: for each $i, \mathbf{M}_{i}$ refines $\mathbf{A}_{i}$. So, the message sent to each receiver does convey a command to that agent. An action-based analysis of the calls could be given simply by taking into account the identity of each receiver: $m_{1}$ means 'do $a_{1}$ !' to one receiver and 'do $a_{2}$ !' to the other receiver. Because this seems more like a difference in dialect than a difference in force, we have an action-based analysis.

While we have argued that both cases in (8) can be given an action-based analysis, we can make the difference between the two precise in the present framework with the help of some new definitions.

- $a_{i}^{m}:=\left\{a \in A\right.$ : for some $\left.c \in R_{i}, \rho_{i}(m, c)=a\right\}$

So: $a_{i}^{m}$ is the set of actions that receiver $i$ takes in some information state in response to hearing message $m$.

- $a^{m}:=\bigcup_{i} a_{i}^{m}$

So: $a_{m}$ is the set of actions that are ever taken by some receiver in response to hearing message $m$.

The two cases in (8) can then be characterized as follows:

a. For every $i$ and $m, a_{i}^{m}$ is a singleton. For every $m, a_{1}^{m}=a_{2}^{m}$. Therefore, for every $m, a^{m}$ is a singleton.

b. For every $i$ and $m, a_{i}^{m}$ is a singleton. For every $m, a_{1}^{m} \neq a_{2}^{m}$. Therefore, for every $m, a^{m}=A$.

Seen in this light, the difference then consists not in whether or not an action-based analysis can be given, but whether one must posit something like dialects to do so. In (8-a), because $a_{1}^{m}=a_{2}^{m}$, a unified action-based analysis can be given. In (8-b), because the action triggered in each agent by each message differs, one must postulate a difference in dialect to provide an action-based analysis.

Finally, we will present two situations - one in which there is only one receiver and one in which the speaker is uncertain about the receivers' identities - in which no action-based analysis can be given according to our criterion.

For the first situation, suppose that there is only one receiver, but it can be in one of two positions. Since moving a couch requires two agents, one can instead imagine that the receiver is leveling a frame hanging on the wall, with input from the sender. We will let $W=\left\{\mathrm{UP}_{l}, \mathrm{UP}_{r}\right.$, Down $_{l}$, Down $\left._{r}\right\}$. Here, the UP states indicate that the left side of the frame is higher than the right; the subscripts indicate whether the receiver is on the left or right side of the frame. As before, $\mathbf{S}$ is the finest partition, i.e. $\mathbf{S}$ has complete information about the state, including the receiver's position. But $\mathbf{R}$ will be the partition that distinguishes between the $l$ and $r$ states (i.e. the receiver only knows its position, but cannot tell which way the frame is tilted). By analogy with the behavior in (8-b), suppose that the sender $\sigma$ sends $m_{1}$ in both UP states and $m_{2}$ in both Down states and that the receiver $\rho$ performs $a_{1}$ in response to $m_{1}$ when it's in position $l, a_{2}$ in response to 
$m_{2}$ in position $l$, and vice versa in position $r$. In this case, because the receiver's response depends not just on the message but also on its position, we have that $\mathbf{M}$ does not refine A and so, by (R), no action-based analysis can be given. This accords well with intuition: $m_{1}$ plausibly means that the frame is tilted up on the left, and $m_{2}$ that it's tilted up to the right. ${ }^{17}$

The second situation is as in the original scenario, but now the speaker does not know which receiver is in the front and which in the back of the couch. So there are four worlds: $W=\left\{\mathrm{UP}_{1}, \mathrm{UP}_{2}\right.$, Down $_{1}$, Down $\left._{2}\right\}$, where the subscripts indicate which receiver is at the front of the couch. $\mathbf{S}$ is the partition distinguishing the up from down states, and $\mathbf{R}_{1}=\mathbf{R}_{2}$ is the partition distinguishing the 1 from 2 states (i.e. the receivers know which position they are in). We have $\vec{a}\left(\mathrm{UP}_{1}\right)=\left\langle a_{1}, a_{2}\right\rangle$ and $\vec{a}\left(\mathrm{UP}_{2}\right)=\left\langle a_{2}, a_{1}\right\rangle$ and vice versa for the down states. In this case, we will have that $\mathbf{M}$ does not refine $\mathbf{A}$, without violating (7) because $\mathbf{M}=\mathbf{S}$ but $\mathbf{S}$ does not refine either $\mathbf{R}_{i}$.

We note that in this modeling situation, no action-based analysis - even based on dialect - can be given. Because the sender lacks information that each receiver has and that each incorporates into its action-selection, it cannot directly command either receiver to take any action. ${ }^{18}$ While we cannot distinguish between a purely indicative meaning (e.g. 'the front of the couch is elevated') and an imperative meaning (e.g. 'lower the front of the couch'), we note that the latter imperative does not qualify as action-based for the present purposes. It presents a group command, specifying a general goal that the receivers are to achieve. But it does not directly cause any particular action on any receiver's part: they still must deliberate and use their information in order to determine which 'concrete' action to undertake. In other words, the general goal under-determines the concrete actions to take, in just the way that $\mathbf{M}$ does not refine $\mathbf{A}$ when no actionbased analysis can be given.

While we have been focused on the analysis of observed call behavior and so omitted any discussion of utility, we also observe that the analyses presented in this section can be made to be optimal by a suitable choice of utility function. For example, if we assume that there is a unique highest-utility action in every cell of $\mathbf{S} \cap \mathbf{R}_{i}$, then if $\mathbf{M}=\mathbf{S}$ and $\rho_{i}$ always chooses that unique action, then this behavior will constitute a strict Nash equilibrium and thus a signaling system in the standard sense.

\section{General Calls and the Informativity Principle}

In the previous section, we addressed property (F1), the assignment of referential meanings. By providing a richer model of the information available to the sender and receivers, we showed that in cases where an action-based analysis cannot be given, either the sender does not have enough messages to convey its information or the sender is not as-ormore-informed than all of the receivers. These conditions are very ecologically plausible, especially when there are multiple receivers. Moving forward, then, we will assume that signals have referential meanings and look for justifications of properties (F2) and (F3): the presence of general calls and competition between calls. We treat these two properties

\footnotetext{
${ }^{17}$ We note that this verdict in one sense agrees and in another disagrees with the discussion of a twoperson version of the game in Zollman [2011], p. 167. He argues that the two-person and three-person case are "essentially equivalent" and that, therefore, the signals should be understood as assertions in both. The present exercise shows that on our analysis of when an action-based analysis can be given, the two cases are not in fact equivalent.

${ }^{18}$ Compare Zollman [2011], p. 167 and the discussion of multiple receivers in the previous section.
} 
together for the following reason: competition among calls requires asymmetric informativity relations between them. When two calls stand in such a relationship, one of them has a strictly more general meaning than the other. Since the call systems we are studying are so simple, this tends to hold when the more general call is in fact a completely general alert call.

We will proceed as follows. First, we will discuss some general worries about the postulation of call systems with general calls and competition between them. In particular, we will motivate the need for analyses where the Informativity Principle in (1) is an optional or defeasible rule. Against that backdrop, we present an initial worry that the presence of an optional competition principle cannot be an evolutionarily stable situation. Then, we present a simple model which allows us to develop a formal proof that the worry is well-founded: under simple assumptions, the only evolutionarily stable situation is obligatory competition. We explore variants of the basic game numerically, and find the same result to hold. ${ }^{19}$ We then discuss the empirical and theoretical consequences of these result, including a discussion of other possible mechanisms that could justify the Informativity Principle.

\subsection{Two Objections to Competition-Based Analyses}

We seek to justify positing call systems with the following features: there are two calls $S$ and $S^{\prime}$, with $S$ having a strictly more general meaning than $S^{\prime}$; by the Informativity Principle (1), listeners infer that an utterance of $S$ excludes the truth of $S^{\prime}$ (and similarly, speakers do not produce $S$ in cases where $S^{\prime}$ applies). There are two objections to the evolutionary stability of systems of this kind: (i) interpretations, not meanings, are fitnessdetermining, and (ii) general calls are unstable. We elaborate each in turn.

As alluded to before, the first objection has been articulated clearly by Jäger [2016] (p. 114): "Meanings are conceptualized as actions of the receiver which induce different fitness values for both sender and receiver. So they correspond to 'interpretations' rather than 'meanings' if we draw a distinction between semantics and pragmatics. Abstract meanings, however, being abstract, are not directly relevant for fitness. This begs the question how they - and therefore the distinction between semantics and pragmaticscould have evolved in the first place in connection with innate signaling systems." In other words: abstract meanings are not selected for; any pressure on the evolution of meanings is indirect, reflecting the actions they cause. But, under an obligatory form of the Informativity Principle, the effects of a general call will look just like those of an enriched call.

While it's true that the net effects of meanings are evaluated for fitness in terms of actions, it does not follow that the mental representation of meanings is in terms of actions. That is to say: a package that posits abstract meanings plus pragmatic interpretive mechanisms may arise from selection pressures other than on the actions they cause. For example, perceptual pressures may induce general representations, while other pressures cause the development of a reasoning module with the relevant structure. These two modules could then be exapted to the communicative setting to produce systems like the ones described in this paper. We do not mean to endorse this particular pathway, and certainly think that more empirical and modeling work is called for here, but rather merely want to drive home the point: even if the net fitness effect is determined by actions, a

\footnotetext{
${ }^{19}$ Code for the numerical results may be found at https://semanticsarchive.net/Archive/ WZmNmEyN/.
} 
modular system with abstract meanings can still evolve.

The second objection maintains that general calls will not be stable. We can make this worry more precise. ${ }^{20}$ In particular, assume the following. (i) Reactions to calls are deterministic: an $A$ triggers an eagle-appropriate response and a $B$ triggers lookingaround. (ii) In general alerts, monkeys always produce a $B$ call. (iii) In eagle alert situations, they produce the $A$ call in a proportion $0<e<1$ of the cases and the $B$ call in the remainder. (iv) In eagle alerts, the appropriate reaction produces utility $u^{*}$ and the looking-around reaction produces utility $u<u^{*}$. (v) Populations are uniform and differ only in the proportion $e$.

Writing $F\left(e^{*}, e\right)$ for the fitness of a population using strategy $e^{*}$ when it invades a population of monkeys using $e$, we can reason about the case where $e^{*}>e$ as follows. The fitness is identical in non-eagle situations. In eagle alert situations, since $e^{*}>e$, the invading population will produce greater utility in encounters with the native population than the native population will. To see this: the invaders will produce $A$ in a larger proportion of eagle scenarios which, by assumptions (i) and (iv), generates utility $u^{*}>u$. Because of this, we will have $F\left(e^{*}, e\right)>F(e, e)$. Because the invading population does better against the natives than the natives do against themselves, it will eventually take over.

Because the above reasoning holds for any $e^{*}>e$, it appears that $e=1-$ the maximum value - will be the only one that can be stable against invaders. But $e=1$ corresponds to the case where $B$ and $A$ are both specific calls. It appears then, that the presence of a general call cannot be stable.

\subsection{Modeling Results}

In this section, we explore the extent to which the above argument generalizes. We will introduce a general framework for studying communication games of the above kind. After formalizing the above argument, we then study various enrichments of the game (with more states, more senders, etc.) to see whether general calls can be stable in any of these other settings. To foreshadow: we find that overlapping meanings, but not strictly general ones, can be stable. After this exercise, we conclude with discussions of the empirical and theoretical consequences of these results.

\subsubsection{The General Framework}

For the emergence of general calls and competition, our model will be similar to that in the previous section, but will differ in one crucial respect: receiver strategies will be derived from sender strategies, instead of being independent. This is because we are modeling the calls as having referential meanings, i.e. as being (analogous to) sets of states. We also omit the partitions from the previous model here. Because we are explicitly concerned with stability here, we also include a utility function. All told then, our model has the following components:

- A space $W$ of worlds

- A probability distribution $P$ on $W$

- A set of messages $M$

${ }^{20}$ Here we follow the discussion on pp. 197-198 of Schlenker et al. [2016b]. 
- Two agents, with strategies $s_{i}: W \rightarrow \Delta(M)$

- A utility function $u: W \times W \rightarrow \mathbb{R}$.

$u\left(w^{\prime}, w\right)$ represents the utility of thinking that the true world is $w^{\prime}$ when in fact it is $w$. For this reason, we will also sometimes write this as $u\left(w^{\prime} \mid w\right)$.

In this case, a play of the game runs as follows:

1. Nature chooses $w \in W$ with probability $P(w)$

(a) Nature chooses, with equal probability, one of the agents to be sender, and the other receiver

(b) Nature informs the sender of $w$

2. The sender sends a message $m$ with probability $s(m \mid w)$

3. The receiver guesses a state $w^{\prime}$

4. Each agent receives $u\left(w^{\prime} \mid w\right)$

The key missing ingredient here is how the receiver chooses its guess $w^{\prime}$. We will assume he does this by Bayesian inference. For a strategy $s$, we define the receiver-counterpart of $s$-denoted $s_{r}$-as follows.

$$
s_{r}(w \mid m):=\frac{s(m \mid w) P(w)}{s(m)}
$$

We can think of this Bayesian receiver strategy in the following way. When a monkey receives a call, it does not deterministically behave in a certain way. Rather, it 'simulates' what would have caused it to produce that very same call. Having simulated some scenarios that could have caused it to produce the call, it generates a guess about what the state is that caused the sender to send the call. Note that when $s$ is non-trivially probabilistic - as when informativity reasoning is optional - so too will $s_{r}$ be. That is: the receiver produces a distribution over possible states upon hearing a call, instead of a single guess.

To analyze the conditions under which general calls and competition can emerge, we want to (a) exhibit strategies that can be said to embody these features and (b) show that they are evolutionarily stable. Before doing (a), we briefly explain the concept of evolutionary stability.

The key intuition is that a strategy is evolutionarily stable if and only if a population of agents all using that strategy cannot be invaded by a small number of mutants playing a different strategy. They are resistant to invasion. To formalize this, we need to define a function $F\left(s^{\prime} ; s\right)$ capturing the fitness of using strategy $s^{\prime}$ in a population of agents all using $s$. A strategy $s$ is an evolutionarily stable strategy (ESS) if and only if, for all $s^{\prime} \neq s$, either: ${ }^{21}$

- $F(s ; s)>F\left(s^{\prime} ; s\right)$, or

- $F(s ; s)=F\left(s^{\prime} ; s\right)$ and $F\left(s ; s^{\prime}\right)>F\left(s^{\prime} ; s^{\prime}\right)$

\footnotetext{
${ }^{21}$ See, e.g., Weibull [1995] for a reference on evolutionary game theory.
} 
This captures the resistance to invasion idea in the following way. Consider a mutant playing $s^{\prime}$. If the first condition holds, it does worse against the $s$ agents, and so gets driven out of the population. Alternatively, in the second condition, it is just as good as $s$ against the $s$ agents, but the $s$ agents do better against the invading $s^{\prime}$ agents, and so it also gets driven out of the population.

To define fitness in the present context, we first define a utility function $U\left(s_{1} ; s_{2}\right)$, which specifies how well $s_{1}$ and $s_{2}$ do when the former is the sender and the latter the receiver. We then take an average of these, capturing that Nature chooses at random who is sender and who is receiver. For the utility $U$, we want to take a weighted average of the payoff to the agents when the receiver guesses that it's world $w^{\prime}$ when the actual world is $w$. The average will be weighted both by how likely it is that the actual world is $w(P(w))$ and by how likely the receiver is to guess that the world is $w^{\prime}$. This latter probability gets decomposed corresponding to how the receiver makes its guess: we look across each message $m$ and see how likely the receiver is to send it in $w\left(s_{1}(m \mid w)\right)$ and then how likely the receiver is to guess $w^{\prime}$ in response to $m\left(s_{2_{r}}\left(w^{\prime} \mid m\right)\right)$. All told, we have:

$$
U\left(s_{1} ; s_{2}\right)=\sum_{w} P(w) \sum_{w^{\prime}} u\left(w^{\prime} \mid w\right) \sum_{m} s_{1}(m \mid w) s_{2_{r}}\left(w^{\prime} \mid m\right)
$$

From this, we define our fitness function as follows, recalling the assumption that each agent is equally likely to be a sender and a receiver in any encounter:

$$
F\left(s_{1} ; s_{2}\right)=\frac{1}{2}\left(U\left(s_{1} ; s_{2}\right)+U\left(s_{2} ; s_{1}\right)\right)
$$

More exact formulations of both functions can be found in the Appendix, where we use them to prove our main theorem, which will be stated in the following section.

\subsubsection{Incorporating General Calls}

In this section, we instantiate the general framework in a way that meets requirement (a) above. We make simple choices for the various parameters of a model and show what a strategy with a general call looks like. These strategies capture the general idea that a general call applies in all kinds of situations, including ones where a more specific call also applies. We then show that in order for more general calls with optional informativity to be stable, there must be a limited number of messages.

Initially, we will assume that $W$ contains two worlds- $E$ (for eagle) and $L$ (for leopard) - and that the agents only have two messages: $m_{1}$ and $m_{2}$. For present purposes, we assume that $m_{1}$ only gets sent in $E$. But, we also want to allow for $m_{2}$ to also be sent in $E$ as well as in $L$ (and, in the following, other states). We will capture this with a competition parameter $\alpha$ : the probability that $m_{1}$ gets sent in $E$. Correspondingly, $1-\alpha$ is the probability that $m_{2}$ gets sent in $E$. Thus, we will denote entire strategies by $\alpha$, which is the only parameter of variation among them. Schematically, the strategies are:

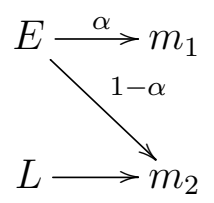

In these strategies, $m_{1}$ means that there is an eagle, while $m_{2}$ means something like there is an alert. When $\alpha=1, m_{2}$ never gets sent in $E$ states. In this sense, it gets 'enriched' 
by systematic competition to mean that there is a non-eagle alert. In many environments where monkeys live, where there is a dominant aerial and a dominant terrestrial predator, $m_{2}$ can thus be taken to indicate the presence of the terrestrial predator.

This model also makes vivid some of the worries discussed in Section 3.1. In particular, the conceptual worry against obligatory competition can be interpreted as asking what would distinguish the $\alpha=1$ case from a case where $m_{2}$ just means leopard. In this model, nothing would distinguish them behaviorally, so appeals to things like natural concepts are the only possible rejoinder. Similarly, the initial worry against optional competition motivates the precise question: is $\alpha=1$ not the only evolutionarily stable strategy in this game?

Before proceeding, we pause for a brief comment on the interpretation of the competition parameter $\alpha$. We are thinking of this parameter as capturing a descriptive generalization, not the implementation of an algorithm. That is to say: an agent playing strategy $\alpha$ uses the general call in a proportion $1-\alpha$ of the cases in which it could have used the more specific call. At our level of modeling, we cannot say exactly which factors influence that choice. Rather, whatever factors influence the choice of call in a given state give rise to behavior that would also be generated by an optional Informativity Principle. On this interpretation, $\alpha$ does not represent a 'coin flip' inside the agent's head when it encounters an eagle. Similarly, $1-\alpha$ should not be interpreted as mere noise, either in production or perception. Were noise in production at issue, one would also expect mis-uses of $m_{1}$ in $L$ situations, which we do not have. Were noise in identifying eagle situations at issue, symmetry considerations would lead one to expect that $L$ situations would also occasionally be mis-identified as $E$ situations and cause signaling behavior that we do not have in the model.

\subsubsection{Results}

Our first result depends on two further simplifying assumptions. First, we restrict the base utility function $u(\cdot, \cdot)$ to those that we will call separating: $u\left(w, w^{\prime}\right)$ always equals the Dirac delta function $\delta\left(w, w^{\prime}\right)$, which is equal to 1 if $w=w^{\prime}$ and to 0 otherwise. Such a utility function maximizes the importance of information transmission in communication: the agents only get rewarded if they guess the exact state that caused production of a given message. Second, we will assume that all states are equiprobable. Both assumptions are common in the signaling games literature. ${ }^{22}$ With these assumptions in place, we can state a preliminary negative result.

If there are two equiprobable states and $u$ is separating, then $\alpha=1$ is the only evolutionarily stable state. ${ }^{23}$

This result provides further vindication for the worries in Section 3.1: we have another situation in which only full informativity $(\alpha=1)$ can be stable. ${ }^{24}$ This raises the question: can optional informativity $(\alpha<1)$ be stable when any of the assumptions that we've made are relaxed?

\footnotetext{
${ }^{22}$ In some cases, one or both are even taken to be definitional of a signaling game. See Wärneryd [1993], Pawlowitsch [2008]. Skyrms [2010], among others, studies signaling games without these restrictions.

${ }^{23}$ We note that the equiprobability assumption is essential: for instance, $\alpha=1 / 2$ can be stable if $P(E)>8 / 9$.

${ }^{24}$ Proof sketch: One can verify that $F(\alpha, \alpha)=1 /(2-\alpha)$ and that $F(\alpha, 1)=\left(5-\alpha^{2}\right) / 4(2-\alpha)$. This yields that $F(\alpha, \alpha)>F(\alpha, 1)$ if and only if $1>\left(5-\alpha^{2}\right) / 4$, which never holds since $\alpha \leqslant 1$.
} 
More worlds than messages. Now, we consider the case where $|W|>|M|$. For concreteness, we fixed $|W|=3$ and $|M|=2$. Using simulations, we did a grid search through all combinations of $\alpha \in\{0,0.25,0.5,0.75,1\}$ and all distributions on worlds taking probability values in $\{0,02,0.4,0.6,0.8,1\} .{ }^{25}$ As before, every ESS discovered has $\alpha=1$. As expected, many have one message being sent exclusively in one world, and another message exclusively in the other two worlds. There are unusually structured equilibria, e.g.:

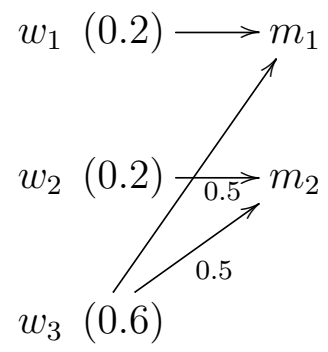

In this equilibrium, there exist overlapping meanings: both $m_{1}$ and $m_{2}$ get sent in state $w_{3}$ (and with equal probability). Nevertheless, neither message is strictly more general than the other, i.e. there are no general calls.

Multiple senders. We also considered the case where there are multiple senders playing the same strategy. In particular, we looked at $|W|=|M|=2$, with two senders who choose a message independently but from the same distribution. We varied the competition as before, and the possible probability distributions on worlds among those with values in $\{0,1 / 6,1 / 3,1 / 2,2 / 3,5 / 6,1\}$. Once again, we found ESSs only with $\alpha=1$, but with partially overlapping messages. For example:

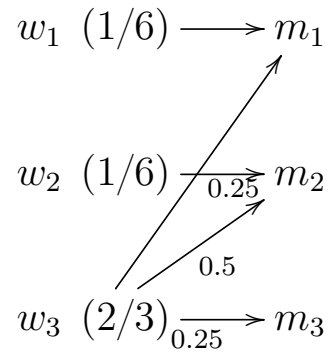

Equilibria like this one allow a group to communicate optimally by implementing a code based on a longer series of bits of information, even though the bits are not independent as in the Zollman games discussed above. It is here the fact that the senders' strategy is not deterministic that makes it possible to encode more bits of information than there are possible messages.

Interim summary. Across many manipulations of parameters of our signaling game, we find that (i) only $\alpha=1$ is ever an evolutionarily stable strategy and that (ii) strictly general calls are not stable. We note that these results depend on simulations where we explored parameter space using grid search, as described above. It is in principle possible that other combinations of parameters than those we explored could yield different results. While we are skeptical, we leave further exploration of the parameter space and the search for analytic versions of our results to future work.

\footnotetext{
${ }^{25}$ Code for running these simulations may be found at https://semanticsarchive.net/Archive/ WZmNmEyN/.
} 


\subsection{Empirical Consequences}

We first assess the consequences of these results for the phenomena analyzed in Section 1: are the analyses there in fact compatible with strengthened meanings and no Informativity reasoning? As alluded to there, one can analyze Putty-nosed monkey pyow-hack sequences in this way. Strengthening a sequence of pyows (general alert) to exclude hack (serious, non-ground, movement-related alert) is unproblematic: the strengthened meaning, even when it occurs in raptor situations, would have a non-serious alert component, which could become true thanks to alarm decay. Similarly, Titi call sequences can also be analyzed with strong meanings and no Informativity reasoning. When $B$ (noteworthy event) appears at the end of sequences, it is compatible with the strengthened meaning of $A$ (serious non-ground alert), because ground alerts will have become non-serious due to alarm decay.

On the contrary, Campbell's calls appear to resist a treatment via strengthened meanings. Strengthening krak (general disturbance) to exclude hok (non-ground disturbance) would roughly have the effect of making it a leopard call. But Schlenker et al. [2014] note that krak is used in the Tai forest in a wider range of contexts than could be explained on such an analysis. In fact, krak is used in contexts where hok could be used and sometimes even in combination with hok. Hence, it seems that $k r a k$ did not acquire a meaning that exclude hok, and this is one of the motivations for a theory based on general meanings and Informativity reasoning. We thus take the difficulty of explaining these call systems to be a limitation of the current model and an avenue for future research.

\subsection{General Consequences}

In the preceding, we have seen that it is indeed difficult to come up with scenarios in which general calls plus optional Informativity reasoning can be evolutionarily stable. There remain, however, other possible routes to arguing for such a package, which we turn to now. Building on the previous discussion of Campbell's monkeys, one can use their call sequences as an empirical argument for the presence of such reasoning and then use theoretical parsimony to extend the style of analysis to other species.

On the modeling side, it should be noted that we made very minimal assumptions about the mechanisms generating behavior consistent with optional competition. Here we offer some initial speculations on more concrete mechanisms that could drive a population towards such behavior. These remarks must remain speculative; but they could be used as springboards for more elaborate modeling.

One option concerns uncertainty on the speaker side. While our model assumes that the Sender receives perfect information about which state obtains, one can also imagine relaxations where the information received can be fuzzy. This could reflect, for example, perceptual limitations. If the Sender is unsure whether there's an eagle or not, it might still want to get the attention of its conspecifics. In situations then where there is in fact an eagle, it might emit a general alert call, relying on the receivers to do the right thing. In other words, there could be situations in which the sender has no more information than the fact that there is a general disturbance, in which case a general call could be useful.

Another option concerns a trade-off between the mental costs of 'storing' certain lexica and engaging in Informativity-based 'reasoning'. This would represent a kind of softening of the argument from non-natural categories above. In particular, it could be that there's a cost to storing certain meanings. This could correlate with a notion of naturalness: 
certain enriched meanings may be so unnatural that they should be excluded as candidate strategies in a signaling game framework. For instance, if the above analysis of Titi calls is right, an analysis without general calls would have to posit: ${ }^{26}$

$$
\llbracket B \rrbracket^{t}=1 \text { iff there is not a serious non-ground alert at } t
$$

Such a meaning seems highly unnatural, because it is intuitively disjunctive. A meaning cost could also reflect informativity, with more 'detailed' meanings being costlier to store. And there could be a 'cost' to carrying out informativity-based reasoning. If these costs are also context-dependent, the balancing of these two costs could lead to a situation where optional Informativity is stable.

\section{Conclusion}

The application of the methods of formal linguistics to monkey alarm calls brings great benefits. But it also raises worries: how does one justify positing properties of alarm calls that appear to only exist in human language and that we cannot test for in the same way that we do for humans? In this paper, we've addressed this worry with respect to three central features of many alarm call systems: (F1) referential meanings, (F2) general calls, and (F3) scalar reasoning between them. We have provided simple models that exhibit (F1). In an extended discussion of (F2) and (F3), we found that many basic models do not allow strategies exhibiting these features to be evolutionarily stable. Future work will have to enrich the models in order to add constraints that can allow us to identify factors that do make such strategies stable. On an empirical level, special attention should be paid to the quantitative evaluation of such cases. ${ }^{27}$ On a theoretical level, it would in the end be important to understand the (possibly very diverse) proximate mechanisms by which meaning enrichment is or is not activated.

\section{Acknowledgments}

We thank Guillame Dezecache and Mélissa Berthet for helpful comments and discussion, as well as two anonymous referees and our handling editor Patrick Georg Grosz for comments that have greatly improved the paper. This research received funding from the European Research Council (ERC) under the European Union's Horizon 2020 research and innovation programme (grant agreement No 788077, Orisem, PI: Schlenker). Research was conducted at Institut d'Etudes Cognitives, Ecole Normale Supérieure - PSL Research University. Institut d'Etudes Cognitives is supported by grant FrontCog ANR17-EURE-0017.

\section{References}

Mélissa Berthet, Christof Neumann, Geoffrey Mesbahi, Cristiane Cäsar, and Klaus Zuberbühler. Contextual encoding in titi monkey alarm call sequences. Behavioral Ecology

\footnotetext{
${ }^{26}$ Because $A$ means there is a serious non-ground alert.

${ }^{27}$ Schlenker et al. [2014] quantitatively compared the uses of krak in eagle situations to the uses of hok in leopard situations. The difference provided a preliminary argument for the general call function of krak.
} 
and Sociobiology, 72(1):1-11, 2018. doi: 10.1007/s00265-017-2424-z.

Mélissa Berthet, Geoffrey Mesbahi, Aude Pajot, Cristiane Cäsar, Christof Neumann, and Klaus Zuberbühler. Titi monkeys combine alarm calls to create probabilistic meaning. Science Advances, 5(5):1-10, 2019. doi: 10.1126/sciadv.aav3991.

Cristiane Cäsar, Klaus Zuberbühler, Robert J. Young, and Richard W. Byrne. Titi monkey call sequences vary with predator location and type. Biology Letters, 9(20130535): 2-5, 2013. doi: 10.1098/rsbl.2013.0535.

Emmanuel Chemla, Isabelle Dautriche, Brian Buccola, and Joël Fagot. Constraints on the lexicons of human languages have cognitive roots present in baboons (Papio papio). Proceedings of the National Academy of Sciences, 116(30):14926-14930, jul 2019. doi: 10.1073/pnas.1907023116. URL http://www.pnas.org/lookup/doi/10.1073/pnas . 1907023116.

Gennaro Chierchia. Logic in Grammar: Polarity, Free Choice, and Intervention. Oxford University Press, 2013.

Gennaro Chierchia, Danny Fox, and Benjamin Spector. Scalar Implicature as a Grammatical Phenomenon. In Claudia Maienborn, Klaus von Heusinger, and Paul Portner, editors, Semantics: An International Handbook of Natural Language Meaning (vol 3), pages 2297-2331. De Gruyter, 2012. doi: 10.1515/9783110253382.2297.

Swan Commier and Mélissa Berthet. Commentary: Titi semantics: Context and meaning in Titi monkey call sequences. Frontiers in Psychology, 10(March):1-2, mar 2019. doi: 10.3389/fpsyg.2019.00512. URL https://www.frontiersin.org/article/10.3389/ fpsyg. 2019.00512/full.

Guillame Dezecache and Melissa Berthet. Working hypotheses on the meaning of general alarm calls. Submitted to Animal Behavior, 2018.

Peter Gärdenfors. Conceptual Spaces: the geometry of thought. The MIT Press, Cambridge, MA, 2000.

John D. Geanakoplos and Heraklis M. Polemarchakis. We can't disagree forever. Journal of Economic Theory, 28(1):192-200, 1982. doi: 10.1016/0022-0531(82)90099-0.

Noah D. Goodman and Andreas Stuhlmüller. Knowledge and Implicature: Modeling Language Understanding as Social Cognition. Topics in Cognitive Science, 5(1):173184, 2013. doi: 10.1111/tops.12007.

Herbert Paul Grice. Logic and Conversation. In Peter Cole and Jerry Morgan, editors, Syntax and Semantics, pages 41-58. Academic Press, New York, 1975.

Jeroen Groenendijk and Martin Stokhof. Studies on the semantics of questions and the pragmatics of answers. Joint phd thesis, Universiteit van Amsterdam, 1984.

Charles L Hamblin. Questions in Montague English. Foundations of Language, 10(1): 41-53, 1973.

Simon M. Huttegger. Evolutionary explanations of imperatives and indicatives. Erkenntnis, 66(3):409-436, 2007. doi: 10.1007/s10670-006-9022-1. 
Gerhard Jäger. Grice, Occam, Darwin. Theoretical Linguistics, 42(1-2):111-115, 2016. doi: 10.1515/tl-2016-0004.

David Lewis. Convention. Blackwell, 1969.

Joseph M. Macedonia and Christopher S. Evans. Variation among Mammalian Alarm Call Systems and the Problem of Meaning in Animal Signals. Ethology, 93(3):177-197, 1993. doi: 10.1111/j.1439-0310.1993.tb00988.x.

Karim Ouattara, Alban Lemasson, and Klaus Zuberbühler. Campbell's monkeys concatenate vocalizations into context-specific call sequences. Proceedings of the National Academy of Sciences, 106(51):22026-22031, 2009. doi: 10.1073/pnas.0908118106.

Eric Pacuit and Olivier Roy. Epistemic Foundations of Game Theory. In Edward N Zalta, editor, The Stanford Encyclopedia of Philosophy. 2015. URL https://plato. stanford.edu/entries/epistemic-game.

Christina Pawlowitsch. Why evolution does not always lead to an optimal signaling system. Games and Economic Behavior, 63(1):203-226, 2008. doi: 10.1016/j.geb.2007. 08.009 .

Andrés Perea. Epistemic Game Theory: Reasoning and Choice. Cambridge University Press, 2012.

Tabitha Price, Philip Wadewitz, Dorothy Cheney, Robert Seyfarth, Kurt Hammerschmidt, and Julia Fischer. Vervets revisited: A quantitative analysis of alarm call structure and context specificity. Scientific Reports, 5:1-11, 2015. doi: 10.1038/srep13220.

Andrea Scarantino. Rethinking Functional Reference. Philosophy of Science, 80(5):10061018, 2013.

Andrea Scarantino and Zanna Clay. Contextually variable signals can be functionally referential. Animal Behaviour, 100:1-8, feb 2015. doi: 10.1016/j.anbehav.2014.08.017.

Philippe Schlenker, Emmanuel Chemla, Kate Arnold, Alban Lemasson, Karim Ouattara, Sumir Keenan, Claudia Stephan, Robin Ryder, and Klaus Zuberbühler. Monkey semantics: two 'dialects' of Campbell's monkey alarm calls. Linguistics and Philosophy, 37:439-501, 2014. doi: 10.1007/s10988-014-9155-7.

Philippe Schlenker, Emmanuel Chemla, Anne M Schel, James Fuller, Jean-Pierre Gautier, Jeremy Kuhn, Dunja Veselinović, Kate Arnold, Cristiane Cäsar, Sumir Keenan, Alban Lemasson, Karim Ouattara, Robin Ryder, and Klaus Zuberbühler. Formal Monkey Linguistics. Theoretical Linguistics, 42:1-90, 2016a. doi: 10.1515/tl-2016-0001.

Philippe Schlenker, Emmanuel Chemla, Anne M Schel, James Fuller, Jean Pierre Gautier, Jeremy Kuhn, Dunja Veselinović, Kate Arnold, Cristiane Cäsar, Sumir Keenan, Alban Lemasson, Karim Ouattara, Robin Ryder, and Klaus Zuberbühler. Formal monkey linguistics: The debate. Theoretical Linguistics, 42(1-2):173-201, 2016b. doi: 10.1515/ tl-2016-0010.

Philippe Schlenker, Emmanuel Chemla, and Klaus Zuberbühler. What Do Monkey Calls Mean? Trends in Cognitive Sciences, 20(12):894-904, 2016c. doi: 10.1016/j.tics.2016. 10.004 . 
Philippe Schlenker, Emmanuel Chemla, Cristiane Cäsar, Robin Ryder, and Klaus Zuberbühler. Titi semantics: Context and meaning in Titi monkey call sequences. Natural Language 85 Linguistic Theory, 35(1):271-298, 2017. doi: 10.1007/s11049-016-9337-9.

Robert M Seyfarth, Dorothy L Cheney, and Peter Marler. Vervet Monkey Alarm Calls: Semantic Communication in a Free-ranging Primate. Animal Behavior, 28:1070-1094, 1980a.

Robert M Seyfarth, Dorothy L Cheney, and Peter Marler. Monkey responses to three different alarm calls: evidence of predator classification and semantic communication. Science, 210(4471):801-803, 1980b. doi: 10.1126/science.7433999.

Brian Skyrms. Signals: Evolution, Learning, and Information. Oxford University Press, 2010.

Shane Steinert-Threlkeld. Compositionality and competition in monkey alert calls. Theoretical Linguistics, 42(1-2):159-171, 2016. doi: 10.1515/tl-2016-0009.

Toshitaka N. Suzuki. Alarm calls evoke a visual search image of a predator in birds. Proceedings of the National Academy of Sciences of the United States of America, 115 (7):1541-1545, 2018. doi: 10.1073/pnas.1718884115.

Karl Wärneryd. Cheap Talk, Coordination, and Evolutionary Stability. Games and Economic Behavior, 5:532-546, 1993. doi: 10.1006/game.1993.1030.

Jürgen Weibull. Evolutionary Game Theory. The MIT Press, Cambridge, MA, 1995.

Brandon C. Wheeler and Julia Fischer. Functionally referential signals: A promising paradigm whose time has passed. Evolutionary Anthropology, 21(5):195-205, 2012. doi: 10.1002/evan.21319.

Kevin J S Zollman. Separating Directives and Assertions Using Simple Signaling Games. The Journal of Philosophy, 108(3):158-169, 2011.

Klaus Zuberbühler. Predator-specific alarm calls in Campbell's monkeys, Cercopithecus campbelli. Behavioral Ecology and Sociobiology, 50(5):414-422, 2001. doi: 10.1007/ s002650100383.

Klaus Zuberbühler, Dorothy L Cheney, and Robert M Seyfarth. Conceptual semantics in a Nonhuman Primate. Journal of Comparative Psychology, 113(1):33-42, 1999. doi: 10.1007/s13398-014-0173-7.2. 